\title{
Risk Factors for Carbapenem- Resistant Pseudomonas aeruginosa, Zhejiang Province, China
}

\author{
Yan-Yan Hu, Jun-Min Cao, Qing Yang, Shi Chen, Huo-Yang Lv, \\ Hong-Wei Zhou, Zuowei Wu, Rong Zhang
}

\begin{abstract}
Carbapenem-resistant Pseudomonas aeruginosa (CRPA) is a public health concern worldwide, but comprehensive analysis of risk factors for CRPA remains limited in China. We conducted a retrospective observational study of carbapenem resistance in 71,880 $P$. aeruginosa isolates collected in Zhejiang Province during 2015-2017. We analyzed risk factors for CRPA, including the type of clinical specimen; the year, season, and region in which it was collected; patient information, including age, whether they were an outpatient or inpatient, and whether inpatients were in the intensive care unit or general ward; and the level of hospital submitting isolates. We found CRPA was more prevalent among isolates from patients $\geq 60$ years of age and in inpatients, especially in intensive care units. In addition, specimen types and seasons in which they were collected were associated with higher rates of CRPA. Our findings can help hospitals reduce the spread of $P$. aeruginosa and optimize antimicrobial drug use.
\end{abstract}

$\mathrm{T}$ he bacterium Pseudomonas aeruginosa is a particularly concerning nosocomial pathogen because of its intrinsic resistance to multiple antimicrobial agents $(1,2)$. In 2016, surveillance of nosocomial infections in China showed $P$. aeruginosa was the fifth most frequently isolated pathogen, accounting for $8.7 \%$ of hospital-acquired infections, and the fourth most common $(8.0 \%)$ in Zhejiang Province $(3,4)$. P. aeruginosa often causes severe infections and results in high rates of illness and death among infected patients (1). A survey in the United States revealed that $P$. aeruginosa was the second-leading cause of nosocomial pneumonia $(14 \%-16 \%)$, third main contributor of urinary tract infections $(7 \%-11 \%)$, and seventh major cause of bloodstream infections $(2 \%-6 \%)(5,6)$.

Author affiliations: Second Affiliated Hospital of Zhejiang University, School of Medicine, Hangzhou, China (Y.-Y. Hu, H.-W. Zhou, R. Zhang); First Affiliated Hospital of Zhejiang University, School of Medicine, Hangzhou (Q. Yang); Zhejiang Provincial Hospital of Traditional Chinese Medicine, Hangzhou (J.-M. Cao, H.-Y. Lv); Hangzhou Third People's Hospital, Hangzhou (S. Chen); lowa State University, Ames, lowa, USA (Z. Wu)

DOI: https://doi.org/10.3201/eid2510.181699
Carbapenems are the most effective antimicrobial agents against severe $P$. aeruginosa nosocomial infections involving bacteria producing cephalosporinase AmpC or extended-spectrum $\beta$-lactamases (7). However, $P$. aeruginosa has become increasingly resistant to carbapenems. A 2016 World Health Organization survey ranked carbapenem-resistant $P$. aeruginosa (CRPA) as the second most critical-priority bacterium among 20 antimicrobial-resistant bacterial species $(8)$.

CHINET surveillance (http://www.chinets.com) revealed that CRPA in Zhejiang Province, China, increased annually from $22 \%$ in 2015 to $38.67 \%$ in 2017 and that Zhejiang had the highest rates of CRPA of all provinces in China in 2017. In addition, Zhejiang reported the local emergence of carbapenem-resistant Klebsiella pneumoniae carbapenemase-producing P. aeruginosa in 2015 (9). Given the clinical importance of CRPA, we analyzed short-term trends and various risk factors related to the occurrence of carbapenem resistance in $P$. aeruginosa in Zhejiang, as well as co-resistance to other commonly used antimicrobial agents.

\section{Materials and Methods}

\section{Bacterial Species and Strain Identification}

We obtained data from the Annual Review of Hospital Infection Resistance Survey in Zhejiang Province, collected during 2015-2017 $(4,10,11)$. Each of the $\geq 78$ secondary or tertiary hospitals enrolled in the surveillance each year (Table 1) imported and shared data of routine antimicrobial susceptibility testing using WHONET 5.6 software (http://www.whonet.org). Enrolled hospitals are distributed in 11 cities of Zhejiang Province: Hangzhou, Huzhou, Jiaxing, Shaoxing, Ningbo, Taizhou, Jinhua, Quzhou, Lishui, Wenzhou, and Zhoushan. Each hospital laboratory cultured isolates on blood agar plates and identified antimicrobial-resistant strains by using matrix-assisted laser desorption/ionization time of flight (MALDI-TOF) mass spectrometry, the VITEK 2 Compact system (bioMérieux, https://www.biomerieux.com), or the Phoenix 100 system (Becton Dickinson, https://www.bd.com). 
Table 1. Pseudomonas aeruginosa isolates obtained from hospitals in Zhejiang Province, China, 2015-2017

\begin{tabular}{|c|c|c|c|c|c|c|c|c|c|}
\hline \multirow[b]{2}{*}{ Year } & \multicolumn{5}{|c|}{ No. hospitals* } & \multirow[b]{2}{*}{ No. isolates } & \multirow[b]{2}{*}{ Isolation rate, $\% \dagger$} & \multirow{2}{*}{$\begin{array}{c}\text { Gram-negative } \\
\text { isolates, \% }\end{array}$} & \multirow{2}{*}{$\begin{array}{l}\text { Imipenem-resistant } \\
\text { isolates, \% }\end{array}$} \\
\hline & Total & $3 \mathrm{~A}$ & $3 \mathrm{~B}$ & $2 \mathrm{~A}$ & $2 B$ & & & & \\
\hline 2015 & 78 & 41 & 23 & 13 & 1 & 22,464 & 8.1 & 11.9 & 35.4 \\
\hline 2016 & 88 & 44 & 23 & 19 & 2 & 24,303 & 8.0 & 12.0 & 37. \\
\hline 2017 & 84 & 41 & 24 & 18 & 1 & 25,113 & 7.8 & 12.0 & 39.1 \\
\hline *Hospi & sificatio & & & & & nission of $\mathrm{Ch}$ & els are given on th & of scores from & $\begin{array}{l}\text { רive evaluation } \\
\text { d supporting facilities. } \\
\text { rehensive evaluation; }\end{array}$ \\
\hline
\end{tabular}

\section{Antimicrobial Susceptibility Testing}

We performed antimicrobial susceptibility testing on 71,880 P. aeruginosa isolates submitted during 20152017. We tested for susceptibility to gentamicin, amikacin, piperacillin/tazobactam, ceftazidime, cefepime, aztreonam, imipenem, meropenem, ciprofloxacin, levofloxacin, colistin, and polymyxin B. We selected these 12 antimicrobial agents because all are used routinely in clinical settings in the province and we could include 1-2 from each antimicrobial category, per guidelines from the Clinical and Laboratory Standards Institute (CLSI; 12). We imported susceptibility data into WHONET, deleted duplicated strains, used only the first isolate from each patient, and interpreted results according to CLSI guidelines (12).

Hospitals prepared isolates for susceptibility testing by using the Kirby-Bauer method and interpreted results manually according to CLSI guidelines (12) or by using broth microdilution for analysis by VITEK 2 or Phoenix 100 automated systems. To ensure comparable susceptibility tests between hospitals, each used the same reference strain, P. aeruginosa ATCC27853, and standardized procedures, following guidelines from the National Health Commission of China. We considered possible inaccuracies of susceptibility tests for colistin and polymyxin B in automated systems, especially by the Kirby-Bauer method, because of poor and slow diffusion in agar plates (13) and applied strict quality control practices by comparing results against our reference strain.

We conducted imipenem susceptibility testing of 71,880 isolates and meropenem susceptibility testing of 26,916 (37.44\%). We used imipenem resistance as an indicator of carbapenem resistance and separately analyzed imipenem-resistant (IMP-R) and imipenem-susceptible (IMP-S) $P$. aeruginosa isolates against the other antimicrobial agents.

\section{Classifications}

We used year as an independent variant for occurrence analysis of IMP-R P. aeruginosa. Then, we calculated other variants by year. For our analysis, we categorized patient age into 6 groups: $0-2,3-9,10-19,20-39,40-59$, and $\geq 60$ years of age. Then we analyzed specific specimen types: blood, sputum, and urine. We analyzed outpatient and inpatient data and divided inpatients into 2 categories: those in intensive care units (ICUs) and those in standard patient wards (non-ICUs). To assess seasonality of CRPA, we analyzed quarters of the year, January-March, AprilJune, July-September, and October-December.

We grouped hospitals into 4 levels, 3A, 3B, 2A, and $2 \mathrm{~B}$, according to classifications designated by the National Health Commission of China, which classifies hospitals on the basis of the number of beds and scores on a comprehensive evaluation. Class 3 hospitals have $>500$ beds, and class 2 hospitals have 100-499 beds. The National Health Commission grades hospitals using scores from a comprehensive evaluation of the number of departments, staffing levels, management, technical level, work quality, and supporting facilities. Grade A hospitals received $>900$ points; grade B hospitals received 750-899 points.

We grouped geographic regions by city (Figure 1). Then, we analyzed each variant by year (Appendix Figure 1, http:// wwwnc.cdc.gov/EID/article/25/10/18-1699-App1.pdf).

\section{Statistical Analysis}

We analyzed antimicrobial resistance patterns of $P$. aeruginosa isolates exported from WHONET. We used unconditional logistic regression models to estimate odds ratios (ORs) and 95\% CIs for univariable analysis of risk factors associated with IMP-R P. aeruginosa. We used either Pearson $\chi^{2}$ test or Fisher exact test to compare the frequency distribution of categorical variables. For all models, we considered $p<0.05$ statistically significant and then performed 2 -sided probability on those results by using SPSS version 23.0 (IBM, https://www.ibm.com). We classified both intermediate and resistant isolates as IMP-R.

\section{Results}

\section{Surveillance Data}

Approximately 80 hospitals from 11 administrative districts in Zhejiang Province participated in the annual survey of antimicrobial resistance. $P$. aeruginosa was the fourth most frequently isolated nosocomial pathogen identified, accounting for $8.0 \%$ of all bacteria obtained and $12.0 \%$ 


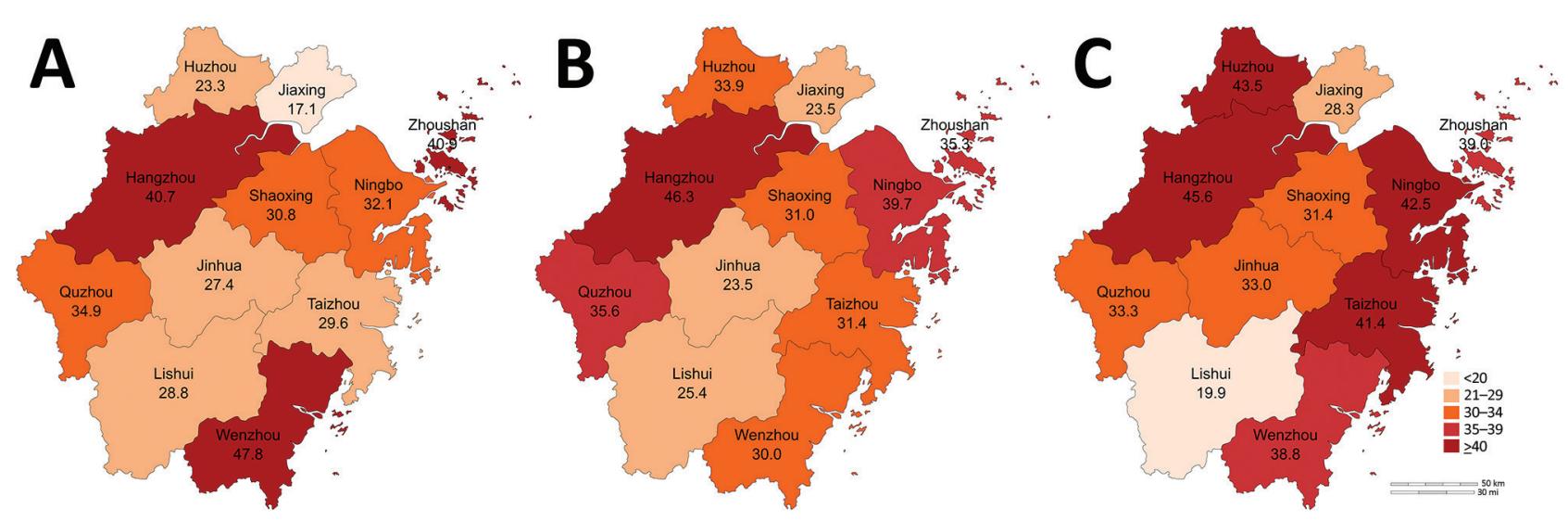

Figure 1. Heatmap of rates of carbapenem-resistant Pseudomonas aeruginosa each year in administrative districts in Zhejiang Province, China. A) 2015; B) 2016; C) 2017.

of gram-negative bacteria collected in Zhejiang. During 2015-2017, hospitals submitted 71,880 P. aeruginosa isolates, $>20,000$ each year; this total is much higher than the numbers analyzed in studies from the United States and Europe $(14,15)$. The large number of isolates provides a strong dataset for our statistical analysis.

We found that 26,789 isolates (37.26\%) were resistant to imipenem. The rate of IMP-R P. aeruginosa was $>35 \%$ in each year and increased gradually during the study period. The meropenem resistance rate of $\approx 29 \%$ was slightly lower than that of imipenem resistance in the 3 years analyzed. In addition, we found that $29.54 \%$ of isolates were resistant to piperacillin/tazobactam and $25.11 \%$ were resistant to cefepime (Table 1; Figure 2; Appendix Table 1).

\section{Correlation of IMP-R P. aeruginosa with Risk Factors}

We examined the correlation between IMP-R P. aerugino$s a$ and risk factors by using OR (Table 2). We investigated quarter of the year, geographic region, patient age, inpatient or outpatient status, and ICU or non-ICU status as risk factors. Our analysis showed that the year isolates were collected had a statistically significant effect on the OR for IMPR P. aeruginosa: OR 1.072 (95\% CI 1.032-1.115) in 2016 compared with 2015 and OR 1.167 (95\% CI 1.124-1.213) for 2017 compared with 2015. Seasonality was also a factor; $P$. aeruginosa isolates collected during January-March,
April-June, and October-December were more likely to be IMP-R than those collected during July-September. We found that the capital of Zhejiang, Hangzhou, as well as Huzhou, Ningbo, Taizhou, Zhoushan, Wenzhou, and Quzhou, had higher IMP-R P. aeruginosa rates than other cities.

Isolates from inpatients had higher rates of imipenem resistance than those from outpatients, and isolates from patients in ICUs were more likely to be IMP-R than those from patients in non-ICU wards. When analyzed for patient age, the highest proportion of resistant isolates were collected from patients $\geq 60$ years of age. We found no statistically significant difference in risk for IMP-R among isolates collected from patients $0-2$ and 3-9 years of age. However, in other age groups, OR increased with age. In addition, we found that isolates from blood and sputum cultures were more likely to be IMP-R than isolates from urine (Table 2).

\section{Antimicrobial Resistance Patterns of $\boldsymbol{P}$. aeruginosa}

Overall, $P$. aeruginosa showed high susceptibility to lipopeptides $(99.07 \%$ to colistin and $98.5 \%$ to polymyxin B) and aminoglycosides (93.06\% to amikacin and $85.88 \%$ to gentamicin) but high resistance to cephalosporins and fluquinolones ( $\approx 20 \%-30 \%$ susceptibility) and aztreonam (35.65\% susceptibility) (Table 3$)$. When we classified isolates into IMP-R and IMP-S groups, we

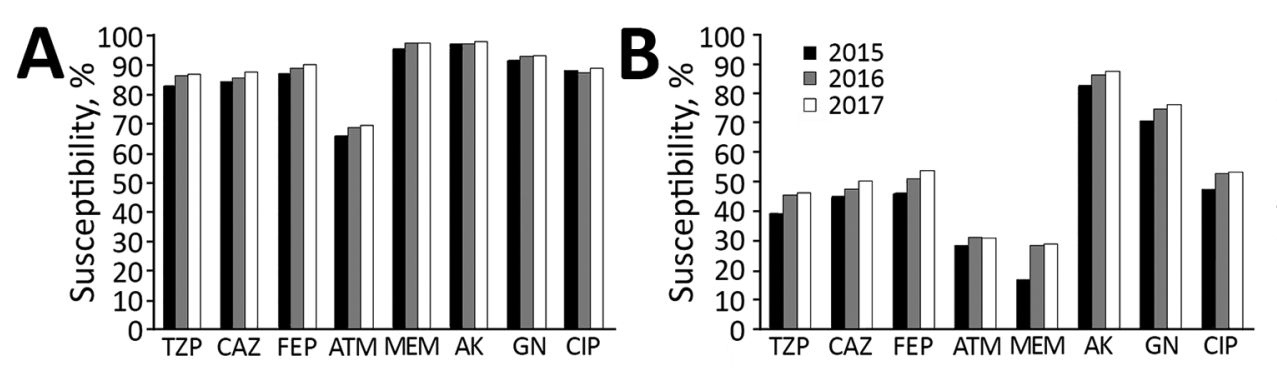

Figure 2. Annual susceptibility rates to antimicrobial agents among imipenem-susceptible (A) and imipenem-resistant (B) Pseudomonas aeruginosa isolates Zhejiang Province, China. AK, amikacin; ATM, aztreonam; CAZ, ceftazidime; CIP, ciprofloxacin; FEP, cefepime; GN, gentamicin; MEM, meropenem; TZP, piperacillin/tazobactam. 
Table 2. Annual odds ratios for risk factors associated with carbapenem-resistant Pseudomonas aeruginosa, Zhejiang Province, China, 2015-2017*

\begin{tabular}{|c|c|c|c|c|c|c|}
\hline \multirow[b]{2}{*}{ Characteristics } & \multicolumn{2}{|l|}{2015} & \multicolumn{2}{|l|}{2016} & \multicolumn{2}{|l|}{2017} \\
\hline & OR $(95 \% \mathrm{Cl})$ & $p$ value & OR $(95 \% \mathrm{Cl})$ & $p$ value & OR $(95 \% \mathrm{Cl})$ & $p$ value \\
\hline \multicolumn{7}{|l|}{ District } \\
\hline Jiaxing & Referent & & Referent & & Referent & \\
\hline Hangzhou & $3.22(2.85-3.63)$ & $<0.001$ & $2.83(2.52-3.19)$ & $<0.001$ & $2.10(1.91-2.31)$ & $<0.001$ \\
\hline Huzhou & $1.42(1.16-1.75)$ & 0.001 & $1.68(1.41-2.00)$ & $<0.001$ & $1.92(1.55-2.38)$ & $<0.001$ \\
\hline Ningbo & $2.23(1.94-2.56)$ & $<0.001$ & $2.16(1.89-2.47)$ & $<0.001$ & $1.85(1.64-2.07)$ & $<0.001$ \\
\hline Taizhou & $1.97(1.65-2.36)$ & $<0.001$ & $1.50(1.29-1.75)$ & $<0.001$ & $1.77(1.51-2.07)$ & $<0.001$ \\
\hline Zhoushan & $3.24(2.63-4.00)$ & $<0.001$ & $1.79(1.46-2.18)$ & $<0.001$ & $1.61(1.31-1.97)$ & $<0.001$ \\
\hline Wenzhou & $4.30(3.75-4.94)$ & $<0.001$ & $1.40(1.18-1.65)$ & $<0.001$ & $1.59(1.40-1.80)$ & $<0.001$ \\
\hline Quzhou & $2.99(2.48-3.61)$ & $<0.001$ & $1.81(1.53-2.14)$ & $<0.001$ & $1.25(1.09-1.45)$ & 0.002 \\
\hline Jinhua & $1.77(1.51-2.08)$ & $<0.001$ & $0.99(0.85-1.15)$ & 0.893 & $1.24(1.09-1.40)$ & 0.001 \\
\hline Shaoxing & $2.09(1.77-2.47)$ & $<0.001$ & $1 . .47(1.26-1.71)$ & $<0.001$ & $1.10(0.96-1.25)$ & 0.165 \\
\hline Lishui & $1.90(1.59-2.27)$ & $<0.001$ & $1.11(0.89-1.39)$ & 0.345 & $0.62(0.49-0.79)$ & $<0.001$ \\
\hline \multicolumn{7}{|l|}{ Specimen type } \\
\hline Urine & Referent & & Referent & & Referent & \\
\hline Blood & $1.23(0.99-1.53)$ & 0.067 & $1.68(1.35-2.08)$ & $<0.001$ & $1.44(1.66-1.77)$ & 0.001 \\
\hline Sputum & $1.87(1.66-2.96)$ & $<0.001$ & $1.97(1.76-2.22)$ & $<0.001$ & $2.13(1.90-2.39)$ & $<0.001$ \\
\hline \multicolumn{7}{|l|}{ Patient age, y } \\
\hline $0-2$ & Referent & & Referent & & Referent & \\
\hline $3-9$ & $0.93(0.58-1.49)$ & 0.764 & $0.83(0.56-1.23)$ & 0.362 & $1.06(0.73-1.54)$ & 0.768 \\
\hline $10-19$ & $1.66(0.99-2.48)$ & 0.055 & $1.23(0.84-1.80)$ & 0.295 & $1.57(1.08-2.29)$ & 0.018 \\
\hline $20-39$ & $3.51(2.48-4.97)$ & $<0.001$ & $2.28(1.70-3.06)$ & $<0.001$ & $2.62(1.95-3.55)$ & $<0.001$ \\
\hline $40-59$ & $3.93(2.82-5.48)$ & $<0.001$ & $2.57(1.95-3.39)$ & $<0.001$ & $3.09(2.33-4.10)$ & $<0.001$ \\
\hline$>60$ & $4.34(3.13-6.02)$ & $<0.001$ & $2.83(2.15-3.71)$ & $<0.001$ & $3.24(2.45-4.27)$ & $<0.001$ \\
\hline \multicolumn{7}{|l|}{ Quarter } \\
\hline Jul-Sep & Referent & & Referent & & Referent & \\
\hline Jan-Mar & $2.11(1.46-3.03)$ & $<0.001$ & $1.30(1.17-1.44)$ & $<0.001$ & $1.90(1.75-2.07)$ & $<0.001$ \\
\hline Apr-Jun & NA & NA & $1.09(0.97-1.22)$ & 0.136 & $1.56(1.42-1.70)$ & $<0.001$ \\
\hline Oct-Dec & NA & NA & $1.28(1.15-1.43)$ & $<0.001$ & $1.21(1.11-1.31)$ & $<0.001$ \\
\hline \multicolumn{7}{|l|}{ Hospital level† } \\
\hline $2 \mathrm{~B}$ & Referent & & Referent & & Referent & \\
\hline $2 \mathrm{~A}$ & $1.36(0.71-2.63)$ & 0.355 & $1.46(0.96-2.20)$ & 0.073 & $1.84(1.11-3.04)$ & 0.016 \\
\hline $3 B$ & $1.13(0.59-2.18)$ & 0.712 & $0.95(0.63-1.44)$ & 0.819 & $1.35(0.82-2.25)$ & 0.239 \\
\hline $3 \mathrm{~A}$ & $1.93(1.01-3.71)$ & 0.044 & $1.10(0.72-1.68)$ & 0.653 & $1.58(0.95-2.63)$ & 3.175 \\
\hline \multicolumn{7}{|l|}{ Type of patient } \\
\hline Outpatient & Referent & & Referent & & Referent & \\
\hline Inpatient, ward & & & & & $1.15(1.01-1.31)$ & 0.039 \\
\hline Non-ICU & Referent & & Referent & & Referent & \\
\hline ICU & $2.60(2.42-2.79)$ & $<0.001$ & $2.66(2.49-2.85)$ & $<0.001$ & $2.57(2.38-2.78)$ & $<0.001$ \\
\hline $\begin{array}{l}\text { *Isolates from patient } \\
\text { OR, odds ratio. } \\
\text { †Hospital classificatio } \\
\text { scores. Comprehensi } \\
\text { Class } 3 \text { hospitals hav } \\
\text { grade A hospitals rec }\end{array}$ & nissing values on the & of & uded in the analysis. & text indice & stical significance. $\mathrm{N}$ & $\begin{array}{l}\text { available; } \\
\text { uation } \\
\text { ng facilities }\end{array}$ \\
\hline
\end{tabular}

found statistically significant differences $(p<0.001)$ in resistance rates between resistant and susceptible isolates for all analyzed antimicrobial drugs except lipopeptides. IMP-R isolates exhibited statistically lower susceptibility than IMP-S isolates to all antimicrobial drugs except the lipopeptides, colistin and polymyxin B. We saw a 2-3-fold difference in $\mathrm{MIC}_{50}$ (MIC needed to inhibit $50 \%$ of cells) between IMP-S isolates and IMP-R isolates. In contrast, for each antimicrobial drug except lipopeptides, most IMP-R strains belonged to the $\mathrm{MIC}_{90}$ group (MIC needed to inhibit $90 \%$ of cells), whereas the IMP-S isolates were more prevalent in the $\mathrm{MIC}_{50}$ group. Similarly, the IMP-R group was highly resistant (25.36\%) to meropenem, but IMP-S group was highly susceptible (96.97\%) (Figure 2).

\section{Discussion}

Carbapenems are the most effective antimicrobial agents against serious infections caused by multidrug-resistant gram-negative bacilli. However, the resistance rate of $P$. aeruginosa to carbapenems has been consistently high (3,16-18). Clarifying resistance trends of CRPA and related risk factors can guide antimicrobial use and selection of effective treatment plans.

In our study, rates of IMP-R P. aeruginosa increased annually and were higher in Zhejiang Province than reported for other provinces in national surveillance through CHINET $(3,17,18)$. For instance, 2017 CHINET surveillance reported national rates of $27.3 \%$ for IMP-R P. aeruginosa and $25.1 \%$ for meropenem-resistant $P$. aeruginosa (18), but in Zhejiang Province the rates were 39.3\% for 
Risk Factors for Carbapenem-Resistant $P$. aeruginosa, China

Table 3. Antimicrobial resistance patterns of imipenem-resistant and imipenem-susceptible Pseudomonas aeruginosa isolates, Zhejiang Province, China, 2015-2017*

\begin{tabular}{|c|c|c|c|c|c|c|c|c|}
\hline \multirow[b]{2}{*}{ Antimicrobial drugs } & \multicolumn{2}{|c|}{ No. isolates (susceptibility rate, \%) } & \multirow[b]{2}{*}{$p$ value } & \multirow{2}{*}{$\begin{array}{c}\text { Total susceptibility } \\
\text { rate, } \%\end{array}$} & \multicolumn{2}{|c|}{$\mathrm{MIC}_{50}, \mu \mathrm{g} / \mathrm{mL}$} & \multicolumn{2}{|c|}{$\mathrm{MIC}_{90}, \mu \mathrm{g} / \mathrm{mL}$} \\
\hline & IMP-S & IMP-R & & & $\mathrm{S}$ & $\mathrm{R}$ & $\mathrm{S}$ & $\mathrm{R}$ \\
\hline Piperacillin/tazobactam & $41,145(85.70)$ & $23,721(44.01)$ & $<0.001$ & 70.46 & 8 & 64 & 64 & 128 \\
\hline Ceftazidime & $30,326(86.26)$ & $18,348(47.93)$ & $<0.001$ & 71.81 & 4 & 16 & 32 & 64 \\
\hline Cefepime & $42,492(89.01)$ & $24,947(50.83)$ & $<0.001$ & 74.89 & 2 & 8 & 16 & 64 \\
\hline Aztreonam & $24,215(68.07)$ & $13,823(30.32)$ & $<0.001$ & 54.35 & 8 & 32 & 32 & 64 \\
\hline Amikacin & 42,106 (97.38) & 24,748 (85.69) & $<0.001$ & 93.06 & 2 & 4 & 8 & 64 \\
\hline Gentamicin & 41,207 (92.80) & 24,618 (74.29) & $<0.001$ & 85.88 & 1 & 2 & 4 & 16 \\
\hline Ciprofloxacin & 42,442 (88.28) & 25,063 (51.64) & $<0.001$ & 74.67 & 0.25 & 1 & 2 & 4 \\
\hline Levofloxacin & 41,982 (89.06) & 24,593 (53.17) & $<0.001$ & 75.80 & 0.5 & 2 & 4 & 8 \\
\hline Meropenem & 17,166 (96.97) & $9,750(25.36)$ & $<0.001$ & 71.03 & 1 & 8 & 1 & 16 \\
\hline Colistin & 1,624 (99.08) & 627 (99.04) & NA & 99.07 & 1 & 1 & 1 & 2 \\
\hline Polymyxin B & $5,012(98.60)$ & 3,746 (98.37) & 0.452 & 98.50 & 1 & 1 & 2 & 2 \\
\hline
\end{tabular}

${ }^{*} \mathrm{MIC}_{50}$ and $\mathrm{MIC}_{90}$ were generated from the minimal inhibitory concentrations of antimicrobial drugs. Bold text indicates $p$ values $<0.05$. IMP-R, imipenemresistant; IMP-S, imipenem-susceptible; NA, not applicable; R, resistant; S, susceptible.

IMP-R and $28.1 \%$ for meropenem-resistant isolates. Both the CHINET surveillance and our data indicated CRPA poses a severe challenge in Zhejiang Province. The slightly lower resistance rate we saw for meropenem could be because we tested fewer isolates for meropenem resistance $(\mathrm{n}=26,916)$ than for imipenem resistance $(\mathrm{n}=71,880)$ or could be the result of other mechanisms, such as mutation or loss of the oprD2 in some isolates (19).

When we examined risk factors, we found that patient type and ward were associated with a higher prevalence of IMP-R $P$. aeruginosa. Inpatients and those admitted to an ICU had higher IMP-R rates than outpatients and those in non-ICU wards, in accordance with previous studies (20), indicating ICU admission is a risk factor for IMP-R $P$. aeruginosa. Patient age also factors into IMP-R P. aerugino$s a$ occurrence in Zhejiang (21), which could be a result of the low immune function of patients $\geq 60$ years of age. We saw an increase in the rate of IMP-R with increased patient age but did not see increased rates for patients $0-2,3-9$, or $10-19$ years of age. However, the IMP-R rate was $>10 \%$ in 2015 and increased to $20.9 \%$ in 2017 in the 10-19-year age group (data not shown), which could signal a potential increasing trend of IMP-R in subsequent years. Further studies with clinical information and data are needed to investigate this issue.

A previous study in India showed that $P$. aeruginosa isolates from sputum and blood samples from patients in the ICU were more resistant than isolates from urine (22). Other studies in China also have observed this discrepancy of $P$. aeruginosa from various specimen types $(16,23)$. We found this observation was true, not only for isolates from patients in the ICU but for all patient isolates included in our study, indicating IMP-R $P$. aeruginosa might be a less likely agent in urinary tract infection.

Previous studies also stated that the occurrence of $P$. aeruginosa infection was associated with seasons $(24,25)$ and that the isolation rate usually was higher in summer than in winter. However, we observed a reverse outcome for IMP-R P. aeruginosa: a higher prevalence in winter than in summer (data not shown). The seasonal effect on IMP-R $P$. aeruginosa rates is unknown, but our finding could potentially inform clinical recommendations.

By OR analysis, we found that IMP-R $P$. aeruginosa was more prevalent in 7 administrative districts: Hangzhou, Huzhou, and Quzhou in the northwest and Ningbo, Taizhou, Zhoushan, and Wenzhou in the southeast of the province. However, we found no statistical differences in IMP-R related to hospital classification in Zhejiang, which is worth noting because patients in class 2 hospitals usually have mild or moderate illnesses and patients in class 3 hospitals have more severe conditions or are immunocompromised and more susceptible to infection. We weighted class 2 hospitals differently than class 3 hospitals in our statistical analysis to account for the difference in patient types. However, because we saw no statistically significant difference in imipenem resistance rates related to the hospital level, we should put the same weight on both classes of hospitals in future analyses.

Although our study showed $P$. aeruginosa was highly resistant to carbapenems and multiple other drugs, it remains highly susceptible to colistin and has some sensitivity to cephalosporins and fluoroquinolones. IMP-R $P$. aeruginosa is most sensitive to colistin in vitro, and colistin is effective against multidrug-resistant $P$. aeruginosa nosocomial infections (26). Despite its strong neurotoxicity and ototoxicity, colistin was reapproved for clinical applications in China in September 2017. However, efficacy of colistin monotherapy has been questioned in clinical trials (27), and colistin should be used in combination with other antimicrobial agents in clinical therapy.

Novel antimicrobial agents approved by the US Food and Drug Administration, such as ceftolozane/tazobactam or ceftazidime/avivactam, could be other treatment options. These drug combinations have good efficacy against CRPA isolates $(28,29)$ but currently are not approved for use in China. Of note, ceftolozane/tazobactam might not be useful against carbapenemase-producing $P$. aeruginosa (30), and prerequisite identification of resistance mechanisms would 
be needed to develop rational antimicrobial drug regimens. In addition, a novel plasmid-mediated colistin-resistant gene, $m c r$, has emerged in Enterobacteriaceae (31-33). To reduce the chances of its dissemination to $P$. aeruginosa under antimicrobial drug selection pressure, clinicians should prioritize colistin only for severe cases of $P$. aeruginosa infection in clinical practice. Because of limitations of susceptibility testing methods (13), MICs for polymyxins might be less reliable in strains with MICs close to the breakpoint. Therefore, clinicians also should choose polymyxin therapies carefully.

Our study had some limitations. We excluded strains without a corresponding field from the classification analysis, such as patient age, patient type, or isolation time, which might have caused a distortion in the resistance rate. A disproportionate number of class 3 to class 2 hospitals participated in the surveillance, and class 2 hospitals inevitably were biased in the statistical antimicrobial resistance rate because they submit fewer isolates. In addition, we could not include therapeutic regimens, patient outcomes, or the molecular mechanisms of resistance for CRPA strains because they were not available, but these measures could inform clinical decisions and should be included in further surveillance studies.

In summary, we conducted a comprehensive analysis of risk factors associated with CRPA in Zhejiang Province, China. We investigated potential risk factors for IMP-R $P$. aeruginosa because Zhejiang Province has higher rates of carbapenem resistance compared with other provinces (34). Our research provides insights into CRPA in China and indicates an imperative for medical institutions in China to strengthen surveillance for this organism.

\section{Acknowledgments}

We thank all the members of the Zhejiang Province Surveillance of Antimicrobial Resistance Program for supplying the data.

This work was funded by the National Natural Science Foundation of China (grant nos. 81501805 and 81772250) and Natural Science Foundation of Zhejiang Province (grant no. LQ16H200002).

Contributions: Y.-Y.H. and R.Z. were involved in the conception and design of the study. J.-M.C., S.C., Q.Y., H.Y.-L., and H.-W.Z. collected the data. Y.-Y.H. and R.Z. analyzed and interpreted the data. Y.-Y.H., Z.W., and R.Z. wrote the manuscript. All authors read and approved the final manuscript.

\section{About the Author}

$\mathrm{Dr}$. $\mathrm{Hu}$ is a clinical laboratory technician at the Second Affiliated Hospital of Zhejiang University, School of Medicine, Zhejiang University, Hangzhou, China. Her primary research interest is antibiotic resistance mechanisms of gram-negative bacteria.

\section{References}

1. Lister PD, Wolter DJ, Hanson ND. Antibacterial-resistant Pseudomonas aeruginosa: clinical impact and complex regulation of chromosomally encoded resistance mechanisms. Clin Microbiol Rev. 2009;22:582-610. https://doi.org/10.1128/ CMR.00040-09

2. Poole K. Pseudomonas aeruginosa: resistance to the max. Front Microbiol. 2011;2:65. https://doi.org/10.3389/fmicb.2011.00065

3. Hu F, Guo Y, Zhu D, Wang F, Jiang X, Xu Y, et al. CHINET surveillance of bacterial resistance across China: report of the results in 2016. Chin J Infect Chemother. 2017;17:481-91. https://doi.org/0.16718/j.1009-7708.2017.05.001

4. Xie X, Yu Y. Annual review of hospital infection resistance survey in Zhejiang Province, 2015 ed. Hangzhou (China): Zhejiang University Press; 2016.

5. Emori TG, Gaynes RP. An overview of nosocomial infections, including the role of the microbiology laboratory. Clin Microbiol Rev. 1993;6:428-42. https://doi.org/10.1128/CMR.6.4.428

6. National Nosocomial Infections Surveillance (NNIS) System report, data summary from October 1986-April 1998, issued June 1998. Am J Infect Control. 1998;26:522-33. https://doi.org/ 10.1016/S0196-6553(98)70026-4

7. Zavascki AP, Carvalhaes CG, Picão RC, Gales AC. Multidrugresistant Pseudomonas aeruginosa and Acinetobacter baumannii: resistance mechanisms and implications for therapy. Expert Rev Anti Infect Ther. 2010;8:71-93. https://doi.org/10.1586/eri.09.108

8. Tacconelli E, Carrara E, Savoldi A, Harbarth S, Mendelson M, Monnet DL, et al.; WHO Pathogens Priority List Working Group. Discovery, research, and development of new antibiotics: the WHO priority list of antibiotic-resistant bacteria and tuberculosis. Lancet Infect Dis. 2018;18:318-27. https://doi.org/10.1016/ S1473-3099(17)30753-3

9. Hu YY, Gu DX, Cai JC, Zhou HW, Zhang R. Emergence of KPC-2-producing Pseudomonas aeruginosa sequence type 463 isolates in Hangzhou, China. Antimicrob Agents Chemother. 2015;59:2914-7. https://doi.org/10.1128/AAC.04903-14

10. Xie XY, Yu YS, Zhang R. Annual review of hospital infection resistance survey in Zhejiang Province, 2017 ed. Hangzhou (China): Zhejiang University Press; 2019

11. Xie X, Yu Y. Annual review of hospital infection resistance survey in Zhejiang Province, 2016 ed. Hangzhou (China): Zhejiang University Press; 2017.

12. Clinical and Laboratory Standards Institute. Performance standards for antimicrobial susceptibility testing; twenty-eighth informational supplement (M100-S28). Wayne (PA): The Institute; 2018.

13. Poirel L, Jayol A, Nordmann P. Polymyxins: antibacterial activity, susceptibility testing, and resistance mechanisms encoded by plasmids or chromosomes. Clin Microbiol Rev. 2017; 30:557-96. https://doi.org/10.1128/CMR.00064-16

14. Woodworth KR, Walters MS, Weiner LM, Edwards J, Brown AC, Huang JY, et al. Vital signs: containment of novel multidrugresistant organisms and resistance mechanisms - United States, 2006-2017. MMWR Morb Mortal Wkly Rep. 2018;67:396-401. https://doi.org/10.15585/mmwr.mm6713e1

15. Kazmierczak KM, de Jonge BLM, Stone GG, Sahm DF. In vitro activity of ceftazidime/avibactam against isolates of Pseudomonas aeruginosa collected in European countries: INFORM global surveillance 2012-15. J Antimicrob Chemother. 2018;73:2777-81. https://doi.org/10.1093/jac/dky267

16. Zhang W, Sun J, Ni Y, Yu Y, Lin J, Yang Q, et al. Resistance profile of Pseudomonas aeruginosa in hospitals across China: the results from CHINET Anitimicrobial Resistance Surveillance Program, 2005-2014 [in Chinese]. Chin J Infect Chemother. 2016;16:141-5.

17. Hu F, Zhu D, Wang F, Jiang X, Xu Y, Zhang X, et al. Report of CHINET Antimicrobial Resistance Surveillance Program in 2015 [in Chinese]. Chin J Infect Chemother. 2016;16:685-94. 
18. Hu F, Guo Y, Zhu D, Wang F, Jiang X, Xu Y, et al. Antimicrobial resistance profile of clinical isolates in hospitals across China: report from the CHINET Surveillance Program, 2017 [in Chinese]. Chin J Infect Chemother. 2018;18:241-51.

19. Shen J, Pan Y, Fang Y. Role of the outer membrane protein OprD2 in carbapenem-resistance mechanisms of Pseudomonas aeruginosa. PLoS One. 2015;10:e0139995. https://doi.org/10.1371/ journal.pone.0139995

20. Raman G, Avendano EE, Chan J, Merchant S, Puzniak L. Risk factors for hospitalized patients with resistant or multidrug-resistant Pseudomonas aeruginosa infections: a systematic review and meta-analysis. Antimicrob Resist Infect Control. 2018;7:79. https://doi.org/10.1186/s13756-018-0370-9

21. Defez C, Fabbro-Peray P, Bouziges N, Gouby A, Mahamat A, Daurès JP, et al. Risk factors for multidrug-resistant Pseudomonas aeruginosa nosocomial infection. J Hosp Infect. 2004;57:209-16. https://doi.org/10.1016/j.jhin.2004.03.022

22. Tennant I, Harding H, Nelson M, Roye-Green K. Microbial isolates from patients in an intensive care unit, and associated risk factors. West Indian Med J. 2005;54:225-31. https://doi.org/10.1590/ S0043-31442005000400003

23. Chen Y, Sun J, Ni Y, Sun Z, Chen Z, Hu Z, et al. 2012 CHINET surveillance of antimicrobial resistance in Pseudomonas aeruginosa in China [in Chinese]. Chin J Infect Chemother. 2015;15:199-203.

24. Richet H. Seasonality in Gram-negative and healthcare-associated infections. Clin Microbiol Infect. 2012;18:934-40. https://doi.org/ 10.1111/j.1469-0691.2012.03954.x

25. Psoter KJ, De Roos AJ, Wakefield J, Mayer J, Rosenfeld M. Season is associated with Pseudomonas aeruginosa acquisition in young children with cystic fibrosis. Clin Microbiol Infect. 2013; 19:E483-9. https://doi.org/10.1111/1469-0691.12272

26. Lee HG, Jang J, Choi JE, Chung DC, Han JW, Woo H, et al. Blood stream infections in patients in the burn intensive care unit. Infect Chemother. 2013;45:194-201. https://doi.org/10.3947/ ic.2013.45.2.194

27. Perez F, Bonomo RA. Evidence to improve the treatment of infections caused by carbapenem-resistant Gram-negative bacteria. Lancet Infect Dis. 2018;18:358-60. https://doi.org/10.1016/ S1473-3099(18)30112-9

28. Spoletini G, Etherington C, Shaw N, Clifton IJ, Denton M, Whitaker P, et al. Use of ceftazidime/avibactam for the treatment of
MDR Pseudomonas aeruginosa and Burkholderia cepacia complex infections in cystic fibrosis: a case series. J Antimicrob Chemother. 2019;74:1425-9. https://doi.org/10.1093/jac/ dky558

29. Fernández-Cruz A, Alba N, Semiglia-Chong MA, Padilla B, Rodríguez-Macías G, Kwon M, et al. A case-control study of real-life experience with ceftolozane-tazobactam in patients with hematologic malignancy and Pseudomonas aeruginosa infection. Antimicrob Agents Chemother. 2019;63:e02340-18.

30. Munita JM, Aitken SL, Miller WR, Perez F, Rosa R, Shimose LA, et al. Multicenter evaluation of ceftolozane/tazobactam for serious infections caused by carbapenem-resistant Pseudomonas aeruginosa. Clin Infect Dis. 2017;65:158-61. https://doi.org/ 10.1093/cid/cix014

31. Liu YY, Wang Y, Walsh TR, Yi LX, Zhang R, Spencer J, et al. Emergence of plasmid-mediated colistin resistance mechanism MCR-1 in animals and human beings in China: a microbiological and molecular biological study. Lancet Infect Dis. 2016;16:161-8. https://doi.org/10.1016/S1473-3099(15)00424-7

32. Wang Y, Tian GB, Zhang R, Shen Y, Tyrrell JM, Huang X, et al. Prevalence, risk factors, outcomes, and molecular epidemiology of mcr-1-positive Enterobacteriaceae in patients and healthy adults from China: an epidemiological and clinical study. Lancet Infect Dis. 2017;17:390-9. https://doi.org/10.1016/ S1473-3099(16)30527-8

33. Tian GB, Doi Y, Shen J, Walsh TR, Wang Y, Zhang R, et al. MCR-1-producing Klebsiella pneumoniae outbreak in China. Lancet Infect Dis. 2017;17:577. https://doi.org/10.1016/ S1473-3099(17)30266-9

34. Zhang R, Liu L, Zhou H, Chan EW, Li J, Fang Y, et al. Nationwide surveillance of clinical carbapenem-resistant Enterobacteriaceae (CRE) strains in China. EBioMedicine. 2017;19:98-106. https://doi.org/10.1016/j.ebiom.2017.04.032

Address for correspondence: Rong Zhang, Zhejiang University, Second Affiliated Hospital of Zhejiang University, School of Medicine, 88 Jiefang Rd, Hangzhou 310009, China; email: zhang-rong@zju.edu. cn; Zuowei Wu, Iowa State University, Department of Veterinary Microbiology and Preventive Medicine, 1113 Vet Med, 1800 Christensen Dr, Ames, IA 50011-1134, USA; email: wuzw@iastate.edu

\section{The Public Health Image Library (PHIL)}

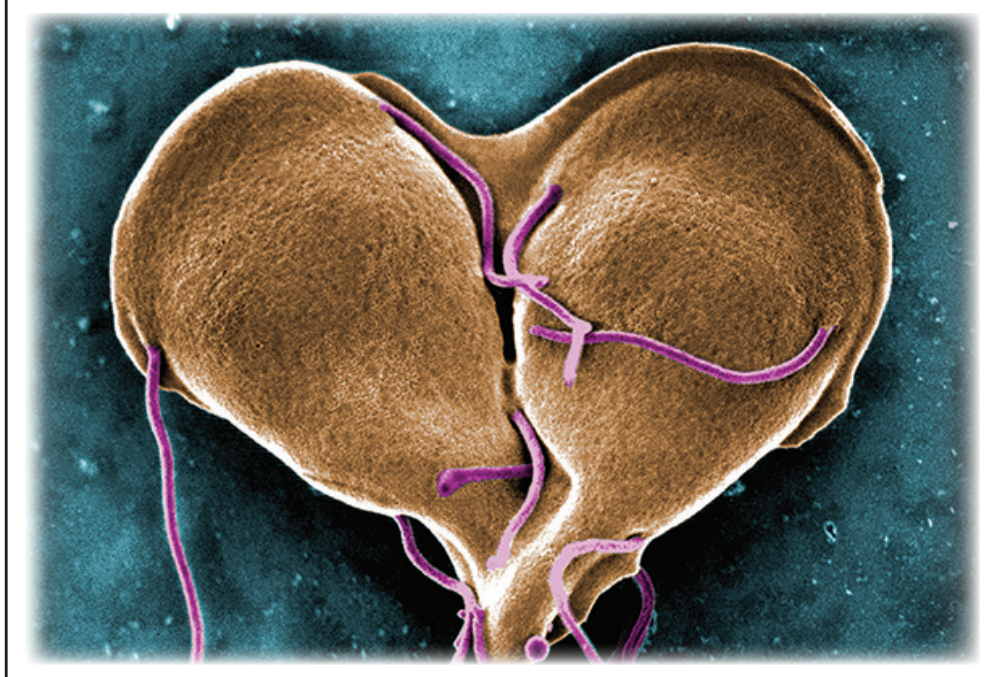

The Public Health Image Library (PHIL), Centers for Disease Control and Prevention, contains thousands of public health-related images, including high-resolution (print quality) photographs, illustrations, and videos.

PHIL collections illustrate current events and articles, supply visual content for health promotion brochures, document the effects of disease, and enhance instructional media.

PHIL images, accessible to PC and Macintosh users, are in the public domain and available without charge.

Visit PHIL at http://phil.cdc.gov/phil 